\title{
Active sensor arrays for damage detection
}

\author{
P. H. Malinowski ${ }^{1, a}$, T. Wandowski ${ }^{1, b}$ and W. M. Ostachowicz $z^{1,2, c}$ \\ ${ }^{1}$ Institute of Fluid-Flow Machinery, Polish Academy of Sciences \\ Fiszera 14, 80-952, Gdansk, Poland \\ ${ }^{2}$ Gdynia Maritime University, Faculty of Navigation \\ Aleja Jana Pawla II 3, 81-345, Gdynia, Poland \\ apmalinowski@imp.gda.pl, btomaszw@imp.gda.pl, ‘wieslaw@imp.gda.pl
}

\author{
Keywords: damage detection, piezoelectric transducers, Structural Health Monitoring, SHM, Lamb \\ waves
}

\begin{abstract}
In presented research a problem that belongs to the Structural Health Monitoring (SHM) topic was investigated. Special arrays of active sensors were used for damage detection. These sensors were piezoelectric transducers. They were attached to specimen under investigation and used to excite and sense guided elastic waves - Lamb waves. Each array comprised of uniquely placed transducers. The total number of transducers was the same for all considered arrays. This ensured that the same number of signals was used to obtain damage information. A numerical algorithm was proposed to process these signals. It was designed to be independent of sensor arrangement so it could be used for all considered arrays. The principal idea behind the algorithm is that obstacles on a wave path cause wave reflection. These reflections are represented in the time signals. The algorithm was used to associate energy of these reflections with a particular area of the investigated specimen. The value of the energy was extracted from all the signals and projected to coordinate system associated with the specimen edges. In order to test and compare proposed arrays artificial defects were introduced to the specimen to model damaged structure. Because the specimen with defect and signal processing algorithm were the same, the only variable that could influence damage detection was the type of the array.

In the investigation damage detection results were obtained for considered arrays. Although the number of sensors were invariable, differences in damage indication exist. This suggest that the type of sensor array should be precisely chosen for a particular application. Even simplest linear array may be sufficient but it depends where we want to apply it.
\end{abstract}

\section{Introduction}

In this paper a research on the SHM topic is presented. A system with sensors that interrogate a structure on-line would increase the safety and reduce the cost connected with unexpected failure. One of the promising methods for such system is based on elastic wave propagation phenomena. The interaction of these waves with defects can bring the diagnostic information. In case of thin walled structures such as aircraft panels these wave are guided by the two parallel surfaces therefore they are called guided waves [1]. However if isotropic material is considered they are called Lamb waves to highlight the Horace Lamb input to the subject [2]. One of the most important features of these waves is that they propagate as symmetric $\left(S_{0}, S_{1}, S_{2}, \ldots\right)$ and antisymmetric $\left(\mathrm{A}_{0}, \mathrm{~A}_{1}, \mathrm{~A}_{2}, \ldots\right)$ modes [3]. The number of both modes is infinite and they are highly dispersive. The dependence of phase velocity on frequency is given be the Rayleigh-Lamb equations [3]:

$$
\frac{\tan (a d)}{\tan (b d)}=-\left[\frac{4 k^{2} a b}{\left(a^{2}-k^{2}\right)^{2}}\right]^{ \pm 1},
$$


where $\mathrm{d}$ is the half of the plate thickness, $\mathrm{k}$ is the wave vector length and $\omega$ is an angular frequency. $a$ and $b$ are defined by

$$
a^{2}=\left(\frac{\omega}{c_{T}}\right)^{2}-k^{2}, b^{2}=\left(\frac{\omega}{c_{L}}\right)^{2}-k^{2}
$$

The exponent +1 is for the symmetric modes, while -1 is for the antisymmetric modes. $c_{\mathrm{T}}$ and $\mathrm{c}_{\mathrm{L}}$ denote the transverse and longitudinal wave velocities, respectively.

There are many way to excite Lamb wave in a structure. Such methods can be listed as angle beam transducers [3], EMATs [4], laser sources [5,6] or piezoelectric elements [7]. By the same means registration can be performed. Nevertheless for an on-line system it is necessary to use small and light sensors that can be permanently attached on or embedded in the structure. Piezoelectric transducers can meet these demands.

Damage detection approaches can be divided in two general groups. One based on concentrated and the second one on distributed arrays. In the first approach such solutions, among others, as phased arrays [7], clock-like array [8] and star-shaped array [9] can be pointed out. As far as distributed arrays are concerned a triangular array [10] and rectangular one [11] are worth mentioning.

In this article an experimental investigation was conducted. Small piezoelectric sensors were used to detect damage in an aluminium alloy panel. These transducers were arranged into three different concentrated arrays and their performance on simulated damage case was tested.

\section{Investigated arrays}

Research reported in this article was focused on three arrays of active sensors (linear, cross and square - Figure 1). Each of considered arrays consisted of nine sensors. Spacing between centres of these elements was equal to $5 \mathrm{~mm}$. Middle sensor of the array was used for Lamb wave excitation. Remaining eight was receiving the reflected waves. The dimensions of the sensors (manufactured by Noliac) were following: $3 \times 3 \times 2 \mathrm{~mm}^{3}$. The goal of the investigation was to employ these arrays for damage detection. In order to do this an object of investigation in the form of an aluminium alloy AA5754 panel was chosen. It's dimensions were following: $1000 \times 1000 \times 1 \mathrm{~mm}^{3}$.

Damage was simulated in the panel by drilling two through thickness holes (Ø6) and introducing two shallow notches, one $10 \mathrm{~mm}$ long and second $184 \mathrm{~mm}$ long. The notches were $0.2 \mathrm{~mm}$ wide. Considered arrays were attached to the panel in such way that the middle sensor of each array was at the centre of the panel. The panel with one of the arrays is presented in Figure 2a. A wax for accelerometer mounting was used to install the sensors, therefore it was possible to use three considered array on the same panel.

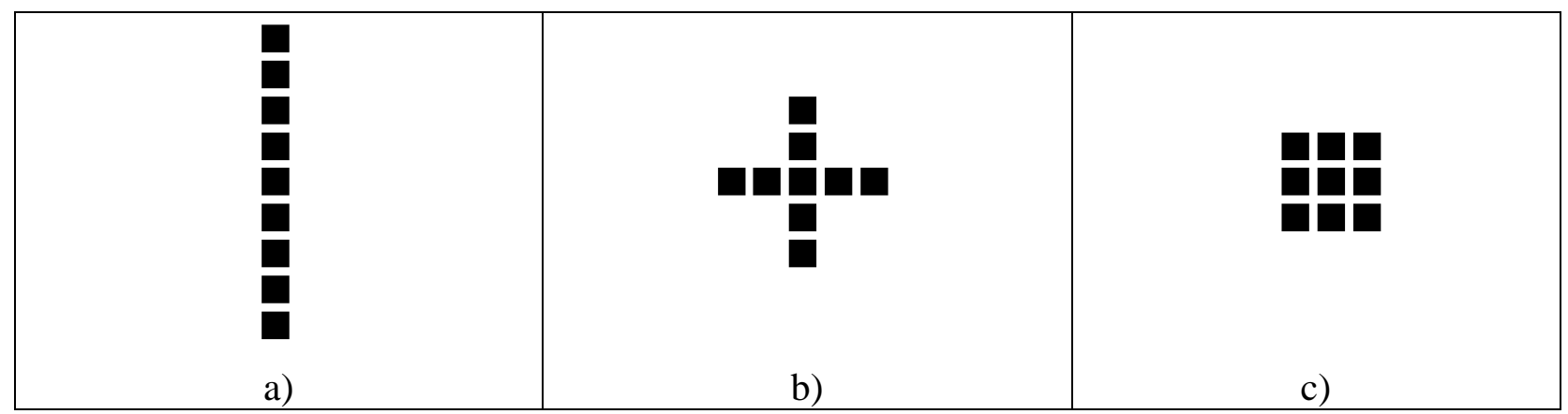

Figure 1. Considered arrays 


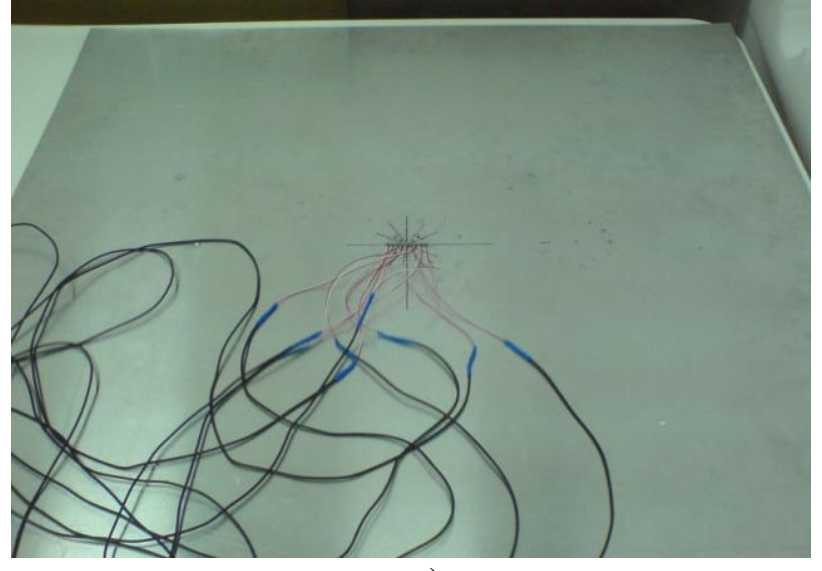

a)

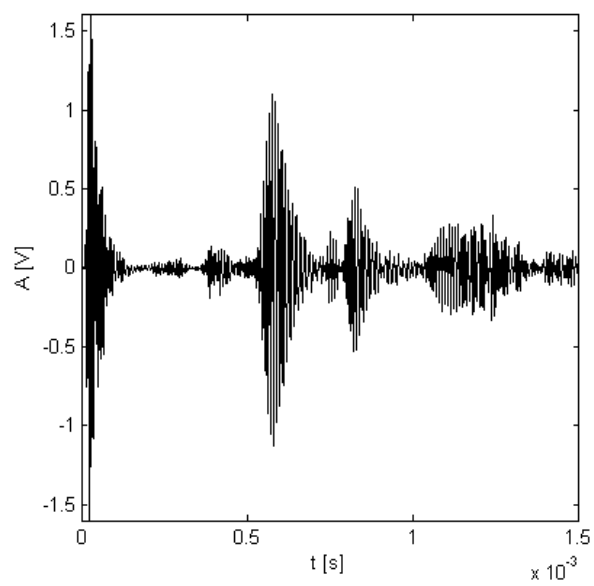

b)

Figure 2. a) Aluminium alloy panel with simulated damage and an array of transducers; b) a part of one of the measured signals

In order to ensure high sensitivity to damage a relatively high frequency waves were used for damage detection. The excitation was chosen in the form of five cycles of sine with Hanning window modulation. The sine frequency was $220 \mathrm{kHz}$. This signal was applied to the middle sensor and registration was performed in remaining eight sensors. Registered signals were processed with special signal processing algorithms in order to obtain information about the panel condition.

\section{Signal processing}

Conducted measurements resulted in eight signals for each considered array. A part of an typical registered signal is depicted in Figure $2 b$. To facilitate the process of obtaining damage information a signal refining procedures were applied. Firstly, signals were shifted by

$$
\Delta t=0.5 \frac{N}{f},
$$

where $\mathrm{N}$ is the number of cycles and $\mathrm{f}$ is the excitation frequency. Value of $\Delta \mathrm{t}$ corresponds to the half of the excitation signal duration, therefore this shifting ensures that in the initial instant $t=0$ the maximum of the excitation is placed. In this research $\mathrm{N}=9$ and $\mathrm{f}=220 \mathrm{kHz}$. Secondly, all registered signals were measured several times and averaged to increase signal to noise ratio. Thirdly, due to imperfect electromechanical shielding a crosstalk occurred resulting in excitation signal present at the beginning of each registered signal. Additionally, because of the fact that the sensors were placed so close to each other wave reflections between them occurred and were registered in the signals. Crosstalk and sensors reflections overlapped in the initial part of the signal making this part useless for damage detection purposes (Figure 2b). In order to remove this part from further analysis the initial parts of the signals lasting $4 \times \Delta \mathrm{t}$ (two times the length of excitation) was set to zero. Fourthly, because the distance from the array to the edges is approximately $0.5 \mathrm{~m}$, the part of the signals representing the response from more than $0.45 \mathrm{~m}$ distance was removed. This ensured that edge reflections do not disturb the damage detection procedure. Lastly, the signals refined in previous steps were normalised by the maximal values. This guaranteed that each of them had an equal input in the damage detection procedure.

\section{Imaging for damage detection}

Signals, that were prepared according to description in the previous section, were used for damage detection. Due to the fact that waves excited in the middle sensor propagate omni-directionally guided by the panel a special mesh of points was introduced. The mesh consisted of concentric 
circles of points (Figure 3). These points were equidistant in radial direction $\Delta \mathrm{r}=10 \mathrm{~mm}$. Also a constant angle step was chosen $\Delta \alpha=5^{\circ}$. The start radius was zero and end radius was equal to $0.5 \mathrm{~m}$ (distance from the middle sensor to the panel edges). The task for the numerical algorithm was to assign to the point of the mesh a numerical value extracted from measured signals. As it can be seen in registered signals (Figure 2b) various wave reflections were registered and the goal is to determine to which simulated defect they correspond.

In order to perform damage detection distances $\left|\mathrm{GP}_{\mathrm{i}}\right|$ and $\left|\mathrm{P}_{\mathrm{i}} \mathrm{R}_{\mathrm{k}}\right|$ were calculated. $\mathrm{G}$ is the point where the generating sensor is placed, $R_{k}$ is the point where one of the receiving sensors is placed $(\mathrm{k}=1,2, \ldots, 8)$ and $\mathrm{P}_{\mathrm{i}}$ is one of the mesh points. These distances were used to cut out a part of the signal registered in $R_{k}$. The cut out part, $F_{n}$, has a length of $l=\Delta r / c_{g}$ and starts at

$$
t_{i k}=\frac{\left|G P_{i}\right|+\left|P_{i} R_{k}\right|}{c_{g}}
$$

where $c_{\mathrm{g}}$ is the wave group velocity obtained in separate measurement. In considered case $c_{g}=2315$ $\mathrm{m} / \mathrm{s}$. It corresponds to the Lamb wave $A_{0}$ mode velocity. $F_{n}$ is discrete so the index takes values $\mathrm{n}=1,2, \ldots, \mathrm{N}, \mathrm{N}$ depends on the length 1 . All the eight registered signals were mapped into point $\mathrm{P}_{\mathrm{i}}$ by summing squared signal from all the receiving sensors $\left(\mathrm{R}_{\mathrm{k}}\right)$ :

$$
M\left(P_{i}\right)=\sum_{k=1}^{K} \sum_{n=1}^{N} F_{n}^{2}
$$

This procedure is repeated for all point $\mathrm{P}_{\mathrm{i}}$ in considered mesh. Such signal processing approach caused that the $\mathrm{M}\left(\mathrm{P}_{\mathrm{i}}\right)$ lies on an ellipsis with loci at $\mathrm{T}$ and $\mathrm{R}_{\mathrm{k}}[12]$.

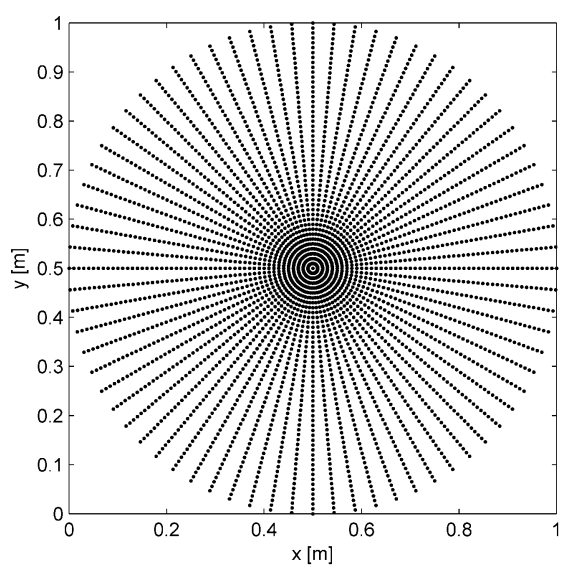

a)

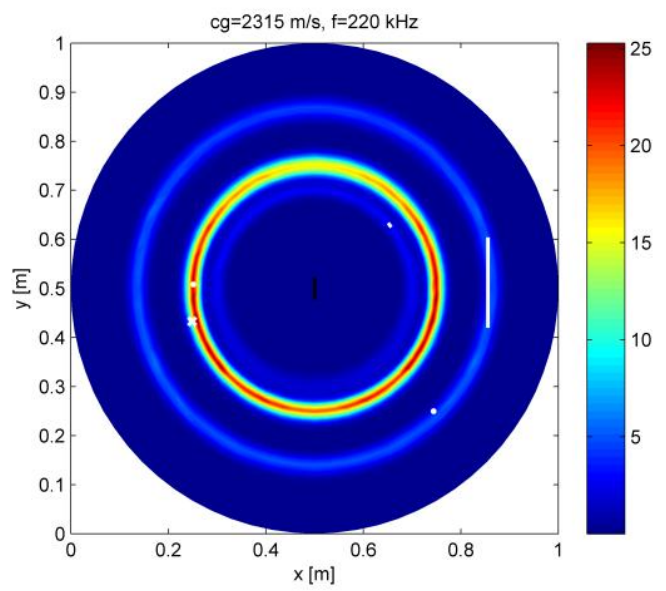

b)

Figure 3. a) mesh of points for damage detection; b) damage detection result for signals gathered by a linear array; white marks indicate the damage position 


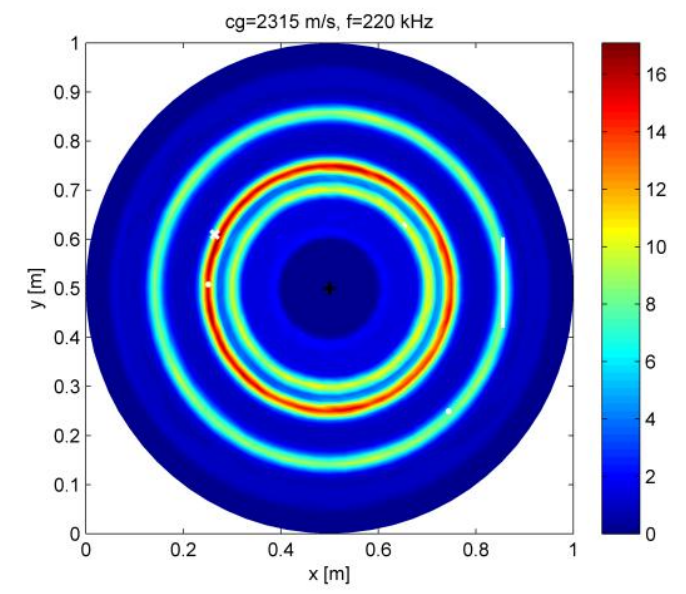

a)

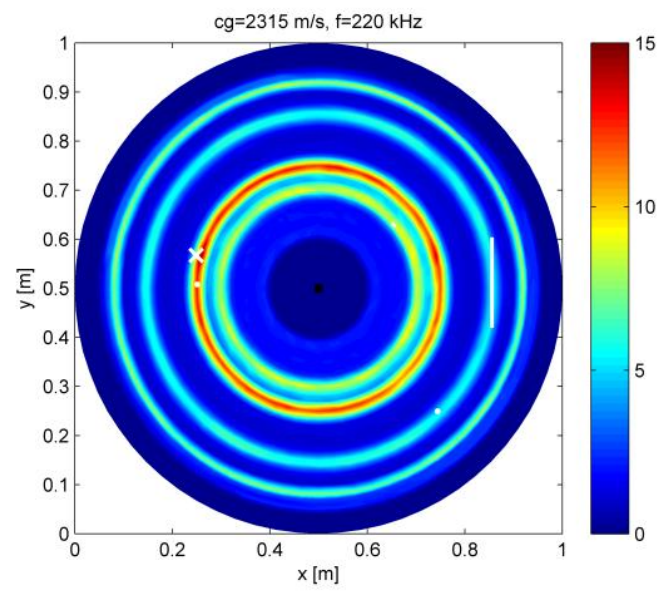

b)

Figure 4. a) damage detection result for signals gathered by a cross array; b) damage detection result for signals gathered by a square array; white marks indicate the damage position

Proposed algorithm returns a $3 \mathrm{D}$ image as a result. The values $\mathrm{M}$ for $\mathrm{P}_{\mathrm{i}}$ points are obtained directly by calculation, while for the intermediate points they are interpolated by the MATLAB plotting function. Figure $3 \mathrm{~b}$, Figure $4 \mathrm{a}$ and Figure $4 \mathrm{~b}$ present the damage detection results for linear, cross and square arrays, respectively. They are investigated thoroughly in subsequent section.

\section{Results and comparison}

Proposed damage detection algorithm was applied to signals gathered by three sensor arrays. Difference between arrays was in individual sensors placement. The individual signals were normalized, therefore the $\mathrm{M}\left(\mathrm{P}_{\mathrm{i}}\right)=1$ was a threshold value. If the presence of a damage is detected value of $\mathrm{M}\left(\mathrm{P}_{\mathrm{i}}\right)$ should be higher than this threshold. The results obtained for all the arrays (Fig.3b, $4 \mathrm{a}$ and $4 \mathrm{~b}$ ) indicate that the amplifications match the damage positions. One can notice high amplitude circles which radii correspond to simulated damage (short notch, long notch and two drilled holes). One circle is noticeable for the long notch and the drilled hole in right lower corner. The reason for this is the fact that the distance from the arrays to this two simulated defects is roughly the same. The linear and cross array detected the simulated defect and nothing else, while the square array results showed an amplification circle with radius larger than for the rest of the defects. Most probably the cause of this additional indication were wires that was laid on the specimen.

Inspecting the values of $\mathrm{M}\left(\mathrm{P}_{\mathrm{i}}\right)$ one can notice that for all three arrays the highest amplification is achieved for the hole on the left hand side. However comparing the amplification value only for this defect, the strongest response (above 25, compare the colorbar) was given by the linear array, lower value is for the cross array (above 17) and the lowest (approx. 15) for the square array. The comparison of the amplification levels for all the arrays is gathered in Table 1. One can notice that the cross and square arrays have the same hierarchy of sensitivity (1. left hand side hole, 2 . short notch, 3. long notch with right hand hole) while for the linear array short notch gives lower response than the long notch with right hand hole. This suggest that the linear array is more sensitive to damage that cause wave reflection perpendicularly to the array.

Table 1. Amplification levels for considered arrays and damage types

\begin{tabular}{|c|c|c|c|}
\hline damage type & linear & cross & square \\
\hline left hand side hole & 25.27 & 17.10 & 15.01 \\
\hline short notch & 3.26 & 11.33 & 9.40 \\
\hline long notch + hole & 5.86 & 10.18 & 6.67 \\
\hline
\end{tabular}

Results obtained for the three arrays (Fig.3b, $4 \mathrm{a}$ and 4b) showed that the information about damage presence can be obtained. Moreover partial localisation was achieved because the radii on 
which the defects lie were found. In order to obtain complete localisation an angular prediction was necessary. To obtain it the areas around the found radii were investigated. The maximal values of $M$ in this areas were calculated. For left hand side hole the maximal value is indicated by white $\times$ in Fig. 3b, 4a and 4b. Unfortunately the position of found maxima differ considerably from the true damage location by 73, 100 and $57 \mathrm{~mm}$ for linear, cross and square array, respectively.

The attempt to obtain angular prediction of the defects failed, therefore only partial information about damage position was obtained. A solution for this could be a phased array processing algorithm [7]. However it needs a great care in selection of sensors spacing and the frequency of excitation.

\section{Summary}

In this paper an experimental study was performed. Its main goal was to compare the performance of the damage detection with three concentrated arrays of sensors. These arrays consisted of nine sensors. The only difference was in the arrangement of the sensors - linear, cross and square. A special numerical algorithm was used to process signals gathered by the sensors and to perform damage detection. A new type of mesh of points was introduced and used with this algorithm. Results of the detection algorithm were compared. In particular it was shown that a linear array is most sensitive to damage that cause wave reflection perpendicular to the array. All the arrays had the highest sensitivity to left hand side hole. However only in the case of a linear array the amplification connected with this damage was so dominant in relation to remaining defects. It was also shown that with the proposed algorithm damage localization is impossible. Phased array algorithm should help to overcome this drawback.

\section{Acknowledgements}

The authors acknowledge the support provided by project MONIT. Pawel Malinowski was also supported by the Polish Ministry of Science and Higher Education (ref. no. N501 335 134).

\section{References}

[1] Z. Su, L. Ye and Y. Lu: Journal of Sound and Vibration Vol. 295 (2006), pp. 753-80.

[2] H. Lamb: Proc. R. Soc. Lond. A Vol. 93 (1917), pp. 114-28.

[3] J. L. Rose: Ultrasonic waves in solid media (Cambridge University Press Cambridge,1999).

[4] P. Wilcox: IEEE T. Ultrason. Ferr. 50 (2003), pp. 699-709.

[5] K. Hongjoon, J. Kyungyoung, S. Minjea and K. Jaeyeol: NDT\&E Int. 39 (2006), pp. 312-19.

[6] K. Hongjoon, J. Kyungyoung, S. Minjea and K. Jaeyeol: Ultrasonics 44 (2006), pp. 1265-68.

[7] V. Giurgiutiu: Structural Health Monitoring with Piezoelectric Wafer Active Sensors (Academic Press, Amsterdam 2008).

[8] W. Ostachowicz, P. Kudela, P. Malinowski and T. Wandowski: Mech. Syst. Signal Pr. 23 (2009), pp. 1805-29.

[9] P. Malinowski, T. Wandowski, I. Trendafilova and W. Ostachowicz W: Struct. Health Monit. 8 (2009), pp. 5-15.

[10] T. Wandowski, P. Malinowski, P. Kudela and W Ostachowicz: Proc.7th Inter. Workshop on Structural Health Monitoring (2009), pp. 2315-22.

[11] W. Qiang and Y. Shenfang : J. Intel. Mat. Syst. Str. 20 (2009), pp. 1663-73.

[12] Y. Lu, L. Ye and Z. Su: Smart Materials and Structures Vol. 15 (2006), pp. 839-49. 